\title{
SLUMP TEST: A NEW EMPIRICAL MODEL FOR HIGH YIELD STRESS MATERIALS
}

\author{
H. KEMER ${ }^{\text {a }}$, R. BOURAS ${ }^{a}$, M. SONEBI $^{\mathrm{b}}$, N. MESBOUA $^{c, \mathrm{~d}^{*}}$, A. BENMOUNAH $^{\mathrm{c}}$ \\ ${ }^{a}$ Laboratory of Experimental and Numerical Modeling of Materials and Structure in Civil Engineering, University Mouloud \\ Mammeri of Tizi Ouzou, Algeria. E-mails: houssam.kemer@ummto.dz, rachid.bouras@ummto.dz \\ ${ }^{\mathrm{b}}$ School of Natural and Built Environment, Queens University of Belfast, Belfast, UK. E-mail: m.sonebi@qub.ac.uk \\ ${ }^{c^{*}}$ Research Unit of Processes, Materials and Environment, University of Boumerdes, Algeria. E-mails: mesbouhnour@hotmail.fr, \\ a.benmounah@univ-boumerdes.dz \\ ${ }^{\mathrm{d}}$ Department of Civil Engineering, faculty of Sciences and applied sciences, University of Bouira, Algeria. E-mail: \\ n.mesboua@univ-bouira.dz
}

Received: 17.07.021 / Accepted: 19.08.2021 / Revised: 21.09.2021 / Available online: 15.12.2021

DOI: 10.2478/jaes-2021-0014

KEY WORDS: High yield stress, Slump test, Rheology, New empirical model.

\begin{abstract}
:
In order to decrease the fitting deviation between rheometrical measurements and empirical parameters using slump test, this paper proposes a new analytical method to evaluate the high yield stress of materials (cement pastes).In doing so, an experimental study was performed for measuring the empirical characteristics by cylindrical mold with different water to binder ratios (w/b) by aiming to increase the yield stress. Parallelly, experimental observations showed clearly the unyielded zone at flow stoppage of high yield stress samples. Based on these results, an idea was formed to applied metallic element at inlet of mold in order to push the spreading of paste. The obtained results of the model proposed in this paper were compared with the yield stress evaluated from shear flow curves using AR2000-rheometer with plate-plate geometry at rate-controlled. In fact, this study demonstrates the applicability and novelty of the present model in the process of relating the empirical parameters to yield stress.
\end{abstract}

\section{INTRODUCTION}

The UN Environment Global Status Report of 2018 projected a doubling of built floor area between now and 2060 (Abergel et al. 2018; Kemer et al. 2021). Concrete taken an important role to this, as largely employed material in construction due to its molding characteristics and high resistance in the hardened state, demanding precision and higher quality control (CQ) standards. These standards must take into consideration not only physical, mechanical, and full life durability properties of that material but also the fresh state (workability) including its transport, placing and final phase of compaction (Kovler and Roussel 2011).In fact, the modern concrete must be sufficiently fluid to fill the formwork in order to cast a given feature (Tattersall and Banfill 1983). In this regards, rheological properties must be assessed scientifically in order to achieve true predictability. On the other hand, concrete is a complex multiphase material (Flatt et al. 2004). In addition to the basic components (cement, sand, aggregates, and water) are added various products such as chemical admixtures (superplasticizers, air entraining agents, retarders, accelerators, etc.) and supplementary cementitious materials (SCMs). The rheological study of fresh concrete is made difficult by the presence of these different phases. Indeed, the latter give the material a significant heterogeneity, leading to the appearance of several types of interactions, including its physical and chemical interactions. Therefore, it seems reasonable to assert that the rheology of concrete is influenced by the behavior of its paste (Benaicha et al. 2015; Bouvet et al. 2010; Legrand 1972;
Sonebi 2010). In fact, the flow of fresh concrete is not that of a pile of pebbles. Rather, it is the paste that (in part) gives concrete its more or less fluid appearance. Cement paste as one of granular suspensions behave like yield stress materials, which flow only after exposed to an optimum shear stress. Additionally, the flow behavior of the latter is governed by the yield stress level. Therefore, determining yield stress is indeed a major issue in several applications (Roussel and Coussot 2005a), including its segregation, formwork pressure, quality of surface and pumping problems (Kwasny et al. 2015) caused by inappropriate evaluation. In the process of identifying value of this interesting parameter, different kinds of apparatus including rheometers are developed in order to evaluate rheological behavior of pastes. Nonetheless, such devices are relatively costly; necessitate a rigorous experimental process and are impractical when used during the concreting phase in the site. Slump and mini-slump tests are, then, used to estimate the workability of concrete and fresh paste mixtures, respectively.

The slump test rests the most common used method to estimate the properties of concrete in fresh state prepared (Noureddine Mesboua et al. 2018; N Mesboua et al. 2021; Yeh 2006, 2007) in the construction site or at the laboratory during the progress of the concreting. Indeed, that is the most basic concrete workability test, with low costs and immediate data. Due to this fact, since 1922, it has been commonly used for workability testing. Descriptively, the mold is a truncated metallic cone opened at both ends and placed on non porous and smooth metallic plate. Gravity causes the paste to slump down while vertically raising the filled cone. 
Several empirical models have been formulated to link the height slump values to both yield stress and density of the material by assuming that the only stress acting on the material is caused by its own weight (Clayton et al. 2003; Murata 1984; Pashias et al. 1996; Saak et al. 2004; Schowalter and Christensen 1998). Furthermore, these studies demonstrate that the geometry and the size of the mold (cone) do not have an impact on the obtained results. For cement paste mixtures characterized by low viscosity or low yield stress values (derived from concretes), Okado et al. (KOKADO and MIYAGAWA 1999) proposed an empirical equation to relate the yield stress to the mold volume (mini-cone) and to the final diameter at flow stoppage of slumped paste named the final spread. The created model is based on the premise that the phenomena are controlled solely by the material's own weight.

Roussel and Coussot (Roussel and Coussot 2005b) were developed these previous theoretical developments, who neglected surface tension, inertia and viscous effects. Therefore, they suggested two analytical solutions for spread $(\mathrm{H}<\mathrm{R})$ and slump flow $(H>R)$ regimes, and demonstrated that these two regimes could be classified as pure shear flow (Eq. 1) and elongational flow (Eq. 2), respectively. These empirical solutions were favorably fitted to experimental data (Roussel et al. 2005) and numerical simulations on cement paste (Roussel and Coussot 2005b). Estelle and Lanos (Estelle and Lanos 2012) also reported a comparison study between yield stress measured from rheometrical vane geometry and slump.

$$
\begin{gathered}
\tau 0=\frac{225 \mathrm{~g} \cdot \rho \cdot V^{2}}{4 \pi^{2} \cdot D f^{5}} \\
\tau 0=\frac{\rho \cdot \mathrm{g} \cdot \mathrm{H}}{\sqrt{3}}
\end{gathered}
$$

where:

$\tau 0, g, \rho, \mathrm{V}, D f$ and $\mathrm{H}$ are: yield stress $(\mathrm{Pa})$, acceleration due to gravity $\left(\mathrm{m} / \mathrm{s}^{2}\right)$, material's density $\left(\mathrm{kg} / \mathrm{m}^{3}\right)$, the mold volume $\left(\mathrm{m}^{3}\right)$, final spread diameter at flow stoppage (m) and final height (m) of sample, respectively.

According to our knowledge and as detailed above, a large divergence was recorded between the empirical equations and the rheometrical measurements in the case of high yield stress materials. Notwithstanding, Alexandre Pierre et al (Pierre et al. 2013) create an intermediate regime in addition to two classical models in order to correctly estimate the yield stress of the Carbopol dispersions whatever the flow regime. That model proved a better correlation with rheometrical measurements at low yield stress values, but close to $100 \mathrm{~Pa}$, the equation has provided a deviation of almost $150 \mathrm{~Pa}$ from the measurements. Consequently and using cement pastes samples, this present paper proposes a new empirical model for high yield stress materials to limit that deviation by applying a metallic element at inlet of cylindrical mold in order to push the unyielded zone and eliminate the peaks and raised edge phenomena (Figure 1) of paste (a small error on the height will induce a significant change in the evaluated yield stress).

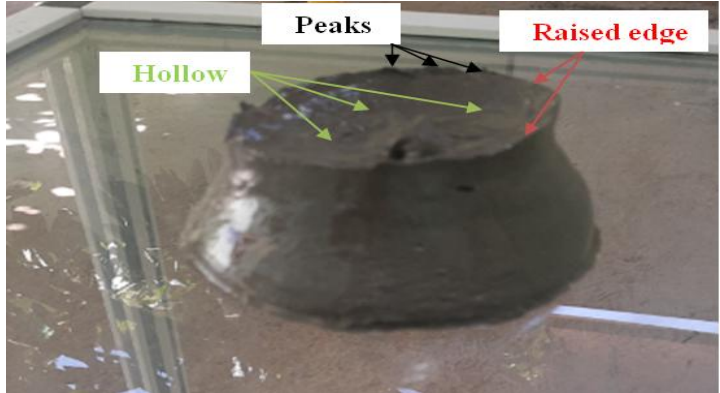

Figure 1 . The cylindrical test of cement past with high yield stress

\section{EXPERIMENTAL PROGRAM}

\subsection{Materials used and samples preparations}

Different tests were conducted on specimens made from (CEM II 42.5) ordinary Portland cement, fresh water; one dosage of MEDAFLOW 30 superplasticizer (1\%) (NF EN 934-2) (Noureddine et al. 2015) with a solid content of $30 \%$ and 1070 $\mathrm{kg} / \mathrm{m}^{3}$ of density was employed and pozzolan powder as mineral admixture (unvarying substitution of $10 \%$ ) with a Blaine surface of $3517 \mathrm{~cm}^{2} / \mathrm{g}$ obtained by Los Angles abrasion machine (Zain et al. 2011). In order to prepare the studied samples, the sustainable cement pastes (SCP) are prepared using various water-to-binder ratios w/b (0.24 to 0.40$)$ by weight; each sample was mixed in a mechanical auto mortar mixer conforming to the specifications of ASTM C 305 (ASTM 2014). All Cement pastes mix designs detailed in Table 1. The mixing protocol

\begin{tabular}{|c|c|c|c|c|}
\hline $\begin{array}{c}\text { Mix } \\
\text { code }\end{array}$ & W/B & $\begin{array}{c}\text { Pozzola } \\
\mathbf{n}(\mathbf{\%})\end{array}$ & $\begin{array}{c}\text { Superplasticiz } \\
\text { er }(\mathbf{\%})\end{array}$ & $\begin{array}{c}\text { Density } \\
\left(\mathbf{k g} / \mathbf{m}^{\mathbf{3}}\right)\end{array}$ \\
\hline CMp1 & 0.40 & 10 & 1.00 & 2004.1 \\
\hline CMp2 & 0.38 & 10 & 1.00 & 2004.2 \\
\hline CMp3 & 0.36 & 10 & 1.00 & 2011.7 \\
\hline CMp4 & 0.34 & 10 & 1.00 & 2013.2 \\
\hline CMp5 & 0.32 & 10 & 1.00 & 2011.9 \\
\hline CMp6 & 0.30 & 10 & 1.00 & 2013.2 \\
\hline CMp7 & 0.28 & 10 & 1.00 & 2014.0 \\
\hline CMp8 & 0.26 & 10 & 1.00 & 2017.3 \\
\hline CMp9 & 0.24 & 10 & 1.00 & 2020.5 \\
\hline
\end{tabular}

detailed in Table 2, was adapted from a previous study.

Table 1. Cement paste mix designs

\begin{tabular}{|c|l|}
\hline Time (min) & \multicolumn{1}{|c|}{ Task } \\
\hline $1: 30$ & $\begin{array}{l}\text { Mix the } 2 / 3 \text { of water }+ \text { total binder at low } \\
\text { speed }\end{array}$ \\
\hline $6: 00$ & Adding 1/3 of water + SP (mix at high speed) \\
\hline $2: 00$ & Pause (stop of mixing) \\
\hline $0: 30$ & remixing \\
\hline $0: 00$ & discharging \\
\hline
\end{tabular}

Table 2. Mixing protocol (Laidani et al. 2020) 


\subsection{Cylindrical tests}

The empirical parameters of cement paste were measured using a cylindrical mold which was made from PVC pipe with a wall thickness of $3 \mathrm{~mm}, 80 \mathrm{~mm}$ in internal diameter and high, open at both ends and sitting on a horizontal surface of glass table $(50 \times 50 \mathrm{~cm})$ and connected with mirror inclined with an angle $\alpha$ of $45^{\circ}$, this mirror recorded by video camera to follow the spread diameter evolutions vs. the w/b ratios as shown in Figure 2 , the mold is filled with cement paste, and lifted vertically, slowly and evenly, because the lifting velocity of the mold has a significant influence on the spread and slump of the mini-slump test for paste with lower yield stress and viscosity. Where a higher lifting velocity may introduce stronger inertial effects, leading to larger spread and slump (Gao and Fourie 2015). After removing the filled mold, the cement paste flowed under gravity on the plate, and finally at flow stoppage, the distance between the original and final position of the centre of the top surface of the sample is measured. This distance is called the slump height (S). The diameter of the slumped sample was measured made in two perpendicular directions and the mean value was reported as the spread $\left(D_{\mathrm{f}}\right)$.

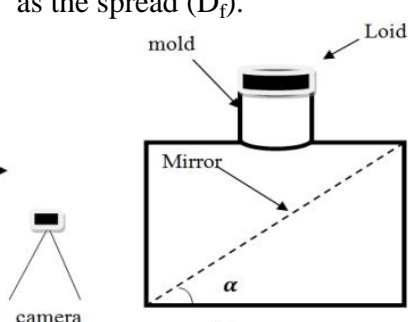

(b)

Figure 2. (a) Equipment for cylindrical test and (b) explicative scheme

\subsection{Rheometrical measurements and testing protocol}

All samples were carried out using AR-2000-rheometer with plate-plate geometry as shown in Figure 3(a) (diameter 14 $\mathrm{mm}$ of the upper plate and constant gap of $1 \mathrm{~mm}$ ) at stress controlled.

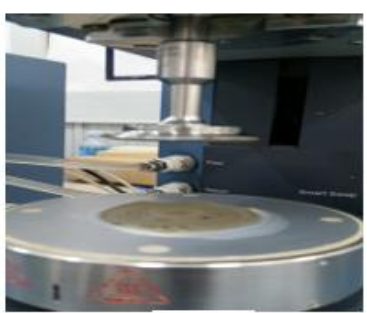

(a)
After mixing, a sample of the predetermined quantity of cement paste was taken immediately into rheometer in order to sandwich the specimen between the upper rotable plate and the lower fixed base plate $($ diameter $=40 \mathrm{~mm})$.

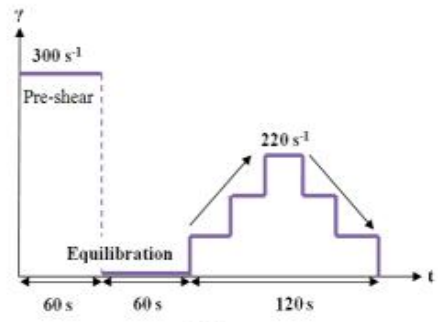

(b)

Figure 3. (a) Plate- plate geometry used for yield stress measurements (upper and lower plates) and (b) stepwise protocol applied in the rheometrical test

For all rheological measurements, the test protocol (Figure 3(b)) has been chosen and applied for all the studied mixtures parallelly with different phases of concreting (Amziane et al. 2005; Roussel 2006; Williams et al. 1999). The samples were maintained at a constant temperature of $20 \pm 1^{\circ} \mathrm{C}$ by an automatic controller during the test. Initially, a presheared at $300 \mathrm{~s}^{-1}$ of the cement pastes for $60 \mathrm{~s}$ and then held at rest period of $60 \mathrm{~s}$. This pre-shear was carried out with an aim of creating an appropriate condition before measuring and insurance of structural breakdown of the paste sample (Nehdi and Rahman 2004). Thereafter, increasing-decreasing shear rate ranging from 0.01 to $220 \mathrm{~s}^{-1}$ was applied through the AR2000 rheometer over $120 \mathrm{~s}$.

\section{EXPERIMENTAL RESULTS}

\section{1. Shear-flow behavior}

Figure 4 shows clearly the shear-flow behavior of the sustainable cement paste dispersion with high yield stress of 300.1Pa. The SCP dispersion is thixotropic (Rachid Bouras et al. 2008) as the up and slope down curves are superimposed
(Figure 4) within the shear rate range investigated. Under the increasing shear rate, a transition to liquid behavior from an elastic solid behavior was also registered. Indeed, there is flow except when the applied shear stress is higher than a threshold value (called yield stress). While, similar trends were registered for other suspensions regardless of the w/b ratios. On the other hand, based on the obtained values of the standard error (SE), it is found that the model of HerschelBulkley (HB) (Eq. 3) fits better $(2<\mathrm{SE}<24.83)$ the experimental data (down slope) for the sustainable cement paste compared to other models such as Bingham and

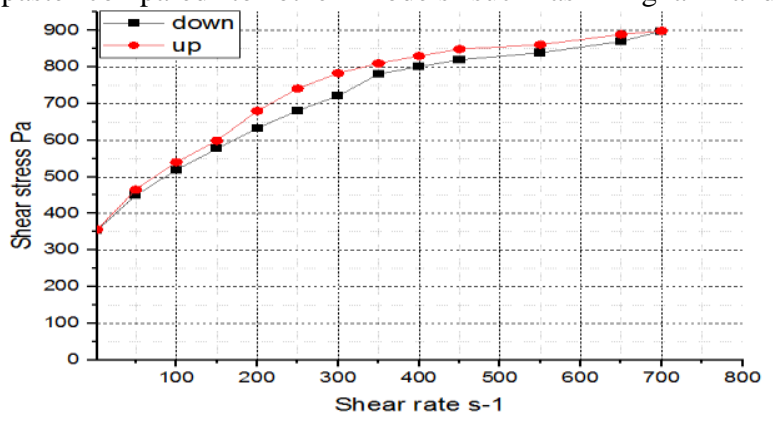

Figure 4. Shear-flow curve of the SCP with $\tau o=300.1 \mathrm{~Pa}$ 
modified Bingham models. This is widely proved in literature (R Bouras 2011; Erzengin et al. 2018; Guo et al. 2017).

$$
\tau=\tau_{0}+\mu \gamma^{\mathrm{n}}
$$

where: $\tau_{0}$ is Herschel yield value $(\mathrm{Pa}), \mu$ is consistency index (Pa.s) and $\mathrm{n}$ is Power law index.

\subsection{Spreading vs. slump behavior}

Using the cylindrical mold with its height to diameter ratio of 1, Figure 5 illustrates clearly some pictures of spreading vs. slump flows tests that were performed using four out of nine different SCP samples. As the yield stress of the SCP increased vs. decreasing of water to binder ratio, the final diameter $(\mathrm{D} f)$ at the same time step decreased and also increasing of the height of final shaping (h) at flow stoppage are registered. On the other hand, Figure 5 illustrates strongly the low spread vs. high shaping state of SCP sample (unyielded zone) at flow stoppage in the case of low w/b ratio as well as the creation of raised edge, peaks and irregular hollow on the top edge of paste after lifting the filled mold as shown in Figure 1. In fact, the measurement of empirical parameters of SCP such as yield stress and height may be hampered as a result of these phenomena taking into consideration their clearly influence in the process of comparing between the yield stress obtained by empirical parameters and rheometrical measurements. Consequently, an idea was formed to applied metallic element. At inlet of mold in order to push the spreading of paste and eliminate those phenomena (seen Figure 6).

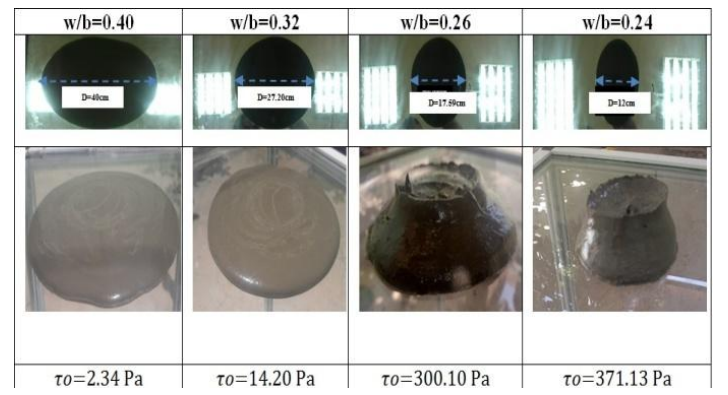

Figure 5. Spreading vs. slump pictures of SCP samples at the

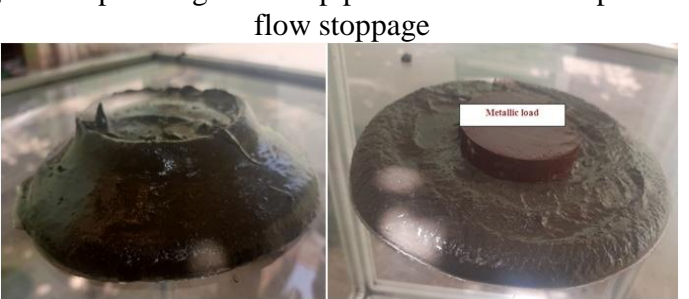

(a)

(b)

Figure 6. High yield stress of SCP (a) without load and (b) under load

\section{PROPOSED OF A NEW ANALYTICAL MODEL}

Using the development of Roussel and Coussot (Roussel and Coussot 2005b) in the case of slump regime when (H>R) with our objective and experimental observation as shown in Figure 7, the flow direction according $\mathrm{z}$ axis only, and the stress in other direction is equal to zero (tangential and radial). At flow stoppage and at any position of $\mathrm{z}$ axis, the results stress is the weight of sample of cement paste and the stress of applied element (W) at inlet of cylindrical mold. The stress of applied element can be expressed as (Eq. 4):

$\mathrm{F}=\frac{W}{S}$

Therefore, the global stress is equal to $-\mathrm{g}[\rho(\mathrm{h}-\mathrm{z})+(\mathrm{M} / \mathrm{s})]$. And that its direction is opposite to the $\mathrm{z}$ axis, which explains the negative sign in front of it.

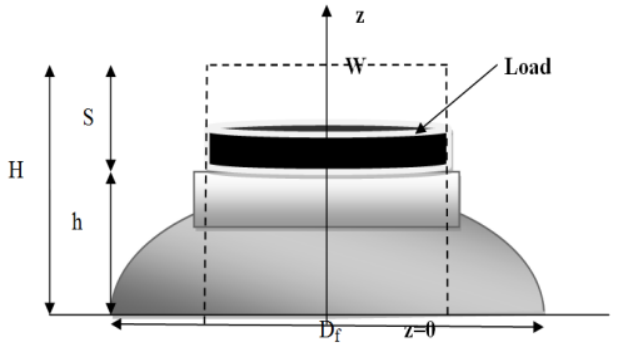

Figure 7. Cylindrical slump at flow stoppage

In which $\rho$ is the material's density expressed in $\mathrm{kg} / \mathrm{m}^{3}, g$ the acceleration due to gravity, $\mathrm{h}$ the height of sample at flow stoppage in $\mathrm{m}$ and $\mathrm{M}$ is the mass of applied element in $\mathrm{kg}$ and $\mathrm{s}$ is the surface of cylindrical load in $\mathrm{m}^{2}$. Therefore, the tensor (T) of results stress at $\mathrm{z}=0$ is (Eq. 5):

$\mathrm{T}=\left[\begin{array}{ccc}\mathrm{g}[\rho \mathrm{h}+(\mathrm{M} / \mathrm{s})] & 0 & 0 \\ 0 & 0 & 0 \\ 0 & 0 & 0\end{array}\right]$

Beyond yielding, the constitutive equation can also be presented in the general form (Eq. 6) (Irgens 2008):

$\mathrm{T}=-\mathrm{PI}+\mathrm{T}^{\prime}$

Where: I is the tensor identity.

The pressure $\mathrm{p}$ at $\mathrm{z}=0$ is given as follow (Roussel and Coussot 2005b) given by Eq. 7:

$\mathrm{P}=-\frac{1}{3} \operatorname{tr}(\mathrm{T})$

Substituting Eq. 7 into Eq. 6, the extra stress tensor is (Eq. 8): $\mathrm{T}^{\prime}=\mathrm{T}-\frac{1}{3} \operatorname{tr}(\mathrm{T}) \mathrm{I}$

Combining Eqs. 5 and (8) gives (Eq. 9):

$\mathrm{T}^{\prime}=\frac{\mathrm{g}[\rho \mathrm{h}+(\mathrm{M} / \mathrm{s})]}{3}\left[\begin{array}{lll}-2 & 0 & 0 \\ 0 & 1 & 0 \\ 0 & 0 & 1\end{array}\right]$

The formula to calculate second invariant of extra-stress tensor is (Eq. 10):

$\mathrm{T}_{\mathrm{II}}=\mathrm{T}_{\mathrm{zz}} \mathrm{T}_{\mathrm{rr}}+\mathrm{T}_{\mathrm{rr}} \mathrm{T} \theta \theta+\mathrm{T}_{\mathrm{zz}} \mathrm{T} \theta \theta-\mathrm{T}^{2}{ }_{12}-\mathrm{T}^{2}{ }_{23}-\mathrm{T}^{2}{ }_{13}$

So, the second invariant ( $\mathrm{T}_{\mathrm{II}}$ ) of extra-stress tensor is calculated as (Eq. 11):

$\mathrm{T}_{\mathrm{II}}=-\frac{\mathrm{g}\left[\rho \mathrm{h}+\frac{M}{S}\right] 2}{3}$

Finally, at low spread vs. high shaping state and at flow stoppage, the yield stress of cement paste depends not only on the height of sample at flow stoppage but also on the applied element at inlet of cylindrical mold. Therefore the yield stress can be calculated by using the following empirical equation (Eq. 12):

$\tau o=\frac{\mathrm{g}\left[\rho \mathrm{h}+\frac{M}{S}\right]}{\sqrt{3}}$ 


\subsection{New model validation}

According to Schlesinger, Buyan et al. (Schlesinger et al. 1974), validation is simply a process by which we can confirm that the conceptual model can be applied or useful when we demonstrate an adequate correspondence between the conceptual results of the model and the actual data if exists or other theorical data. Therefore, using this concept in the process of validation the applicability and novelty of our proposed model is applied. As shown in Figure5 and mentioned above, the yield stress data of the samples obtained from plate-plate measurements vary between 2.34 and $300.10 \mathrm{~Pa}$ depending strongly on the $\mathrm{w} / \mathrm{b}$ ratios of the SCPs. These data are compared with the empirical yield stress values obtained from the cylindrical-slump measurement and calculated with our developed model (Eq. 12), as set out in Figure 8. Comparatively, the solution of the analytical spread model (Eq.1) and slump model (Eq.2) are also reported in that figure (Figure 8).

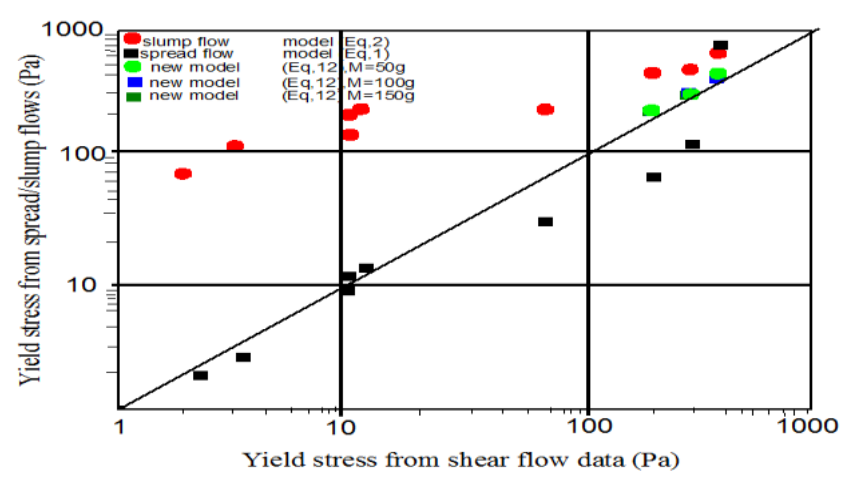

Figure 8. New empirical model (Eq. 12), Roussel's regimes considering slump flow (Eq. 2), and spread flow (Eq. 1) versus the yield stress values calculated from AR 2000-rheometer with the Herschel-Bulkley model

At low yield stress values ranging from 2.34 to $14.20 \mathrm{~Pa}$, we register a higher prediction of Roussel's model (Roussel and Coussot 2005b) (Eq. 1) with yield stress, which provided a better fitting with the values of rheometrical yield stress estimated from the Herschel-Bulkley data. Therefore, above $61.15 \mathrm{~Pa}$, a low spread vs. slump is observed, which corresponds to the elongational regime (Roussel and Coussot 2005b) (Eq. 2). This model proves its limitation by referring to the lower prediction with shear-flow curve data proved by higher deviation of almost $150 \mathrm{~Pa}$. Comparatively, the new model (Eq. 12), which takes into account in the its mathematical equation the applied metallic element on the top edge of sample at flow stoppage, is proved its applicability and novelty based on the good fitting and lower deviation with measurements compared to Eq. 2 and what reported in the literature (Pierre et al. 2013). Furthermore, the proposed model registered low sensibility with varying of applied element of 50,100 and $150 \mathrm{~g}$ for all pastes with w/b rations of $0.24,0.26$ and 0.28 . As shown in Figure 8.

\section{CONCLUSION}

Based on the data reported in this paper regarding the new empirical model of high yield stress using slum test of mixes tested with ranges of w/b from 0.24 to 0.40 , the following conclusions were drawn:

- At low spread compared to high shaping state and at flow stoppage, is not necessarily to limit the flow behavior in a single regime (slump regime), and can lead to another model.

- Consequently, the new proposed model was developed in order to correctly estimate the yield stress of sustainable cement paste by assuring a proper spreading at flow stoppage.

- To do so, the metallic element, applied to push the unyielded zone and eliminate its peaks, raised edge and irregular hollow on the top edge phenomena of paste after lifting the filled mold.

- The Validity of the proposed model was assessed using AR-2000 rheometer -TA instrument data with plate-plate geometry, this proposed new model demonstrates its novelty and applicability.

\section{Reference}

Abergel, T, et al. (2018), 'Global Status Report. Towards a zero-emission, efficient, and resilient buildings and construction sector', Global Alliance for Buildings and Construction. Available online: https://www. worldgbc. org/sites/default/files/2018\% 20GlobalABC\% 20Global\% 20Status\% 20Report (accessed on 19 June 2020).

Amziane, Sofiane, Ferraris, Chiara F, and Koehler, Eric P (2005), 'Measurement of workability of fresh concrete using a mixing truck', Journal of research of the National Institute of Standards and Technology, 110 (1), 55.

ASTM, ASTM (2014), '305-14 Standard practice for mechanical mixing of hydraulic cement pastes and mortars of plastic consistency ASTM C305-14', ASTM Int. West Conshohocken PA.

Benaicha, Mouhcine, et al. (2015), 'Marsh cone coupled to a plexiglas horizontal channel: Rheological characterization of cement grout', Flow Measurement and Instrumentation, 45, 126-34.

Bouras, R (2011), 'Rheology of cement pastes for selfcompacting concrete', (PhD thesis, Mouloud Mammeri University of Tizi Ouzou).

Bouras, Rachid, Chaouche, Mohend, and Kaci, Salah (2008), 'Influence of viscosity-modifying admixtures on the thixotropic behaviour of cement pastes', Applied Rheology, 18 (4), 45604-1-04-8. 
Bouvet, Adrien, et al. (2010), 'The mini-conical slump flow test: Analysis and numerical study', 40 (10), 1517-23.

Clayton, S, Grice, TG, and Boger, DV (2003), 'Analysis of the slump test for on-site yield stress measurement of mineral suspensions', International journal of mineral processing, 70 (1-4), 3-21.

Erzengin, S Gamze, et al. (2018), 'The properties of cement systems superplasticized with methacrylic ester-based polycarboxylates', Construction and Building Materials, 166, 96-109.

Estelle, Patrice and Lanos, Christophe (2012), 'High torque vane rheometer for concrete: principle and validation from rheological measurements', Applied Rheology, 22 (1).

Flatt, Robert J, Martys, Nicos, and Bergström, Lennart (2004), 'The rheology of cementitious materials', MRS bulletin, $29(5), 314-18$.

Gao, Jinglong and Fourie, Andy (2015), 'Using the flume test for yield stress measurement of thickened tailings', Minerals Engineering, 81, 116-27.

Guo, Yiqun, et al. (2017), 'Evaluating the distance between particles in fresh cement paste based on the yield stress and particle size', Construction and building materials, 142, 109-16.

Irgens, Fridtjov (2008), Continuum mechanics (Springer Science \& Business Media)

Kemer, Houssam, et al. (2021), 'Shear-thickening behavior of sustainable cement paste-Controlling physical parameters of new sources of supplementary cementitious materials', 310, 125277.

KOKADO, Takeshi and MIYAGAWA, Toyoaki (1999), 'Study on a method of obtaining rheological coefficients of high-flow concrete from slump flow test', Doboku Gakkai Ronbunshu, 1999 (634), 113-29.

Kovler, Konstantin and Roussel, Nicolas (2011), 'Properties of fresh and hardened concrete', Cement and Concrete Research, 41 (7), 775-92.

Kwasny, Jacek, et al. (2015), 'Influence of rheology on the quality of surface finish of cement-based mortars', Construction and Building Materials, 89, 102-09.

Laidani, Zine El-Abidine, et al. (2020), 'Experimental investigation on effects of calcined bentonite on fresh, strength and durability properties of sustainable selfcompacting concrete', 230, 117062 .

Legrand, Claude (1972), 'Contribution à l'étude de la rhéologie du béton frais', Matériaux et Construction, 5 (5), 275-95.

Mesboua, N, et al. (2021), 'CALCINATED BENTONITE AS SUPPLEMENTARY CEMENTITIOUS MATERIALS IN CEMENT-BASED MORTAR', 11 (1).

Mesboua, Noureddine, Benyounes, Khaled, and Benmounah, Abdelbaki \%J Cogent Engineering (2018), 'Study of the impact of bentonite on the physico-mechanical and flow properties of cement grout', 5 (1), 1446252.

Murata, J (1984), 'Flow and deformation of fresh concrete', Materiaux et Construction, 17 (2), 117-29.

Nehdi, M and Rahman, M-A (2004), 'Estimating rheological properties of cement pastes using various rheological models for different test geometry, gap and surface friction', Cement and concrete research, 34 (11), 1993-2007.

Noureddine, Mesboua, et al. (2015), 'Influence de l'embuvage et de l'élancement des fibres sur les propriétés des mortiers destinés aux bétons de fibres', 2 (3), 145-57.

Pashias, N, et al. (1996), 'A fifty cent rheometer for yield stress measurement', Journal of Rheology, 40 (6), 1179-89.

Pierre, Alexandre, Lanos, Christophe, and Estelle, Patrice (2013), 'Extension of spread-slump formulae for yield stress evaluation', Applied Rheology, 23 (6).

Roussel, N and Coussot, Ph \%J Journal of rheology (2005a), "'Fifty-cent rheometer" for yield stress measurements: from slump to spreading flow', 49 (3), 705-18.

Roussel, N and Coussot, Ph (2005b), '"Fifty-cent rheometer" for yield stress measurements: from slump to spreading flow', Journal of rheology, 49 (3), 705-18.

Roussel, Nicolas (2006), 'A thixotropy model for fresh fluid concretes: theory, validation and applications', Cement and concrete research, 36 (10), 1797-806.

Roussel, Nicolas, Stéfani, Christian, and Leroy, Robert (2005), 'From mini-cone test to Abrams cone test: measurement of cement-based materials yield stress using slump tests', Cement and concrete research, 35 (5), 817-22.

Saak, Aaron W, Jennings, Hamlin M, and Shah, Surendra P (2004), 'A generalized approach for the determination of yield stress by slump and slump flow', Cement and concrete research, 34 (3), 363-71.

Schlesinger, S, et al. (1974), 'Developing standard procedures for simulation validation and verification(autopilot, atmosphere and transportation models)', Summer Computer Simulation Conference, Houston, Tex, 927-33.

Schowalter, WR and Christensen, G (1998), 'Toward a rationalization of the slump test for fresh concrete: comparisons of calculations and experiments', Journal of Rheology, 42 (4), 865-70.

Sonebi, Mohammed (2010), 'Optimization of cement grouts containing silica fume and viscosity modifying admixture', Journal of materials in civil engineering, 22 (4), 332-42.

Tattersall, Geoffrey Howarth and Banfill, Phillip FG (1983), The rheology of fresh concrete.

Williams, David A, Saak, Aaron W, and Jennings, Hamlin M (1999), 'The influence of mixing on the rheology of fresh cement paste', Cement and concrete research, 29 (9), 1491-96.

Yeh, I-Cheng (2006), 'Exploring concrete slump model using artificial neural networks', Journal of Computing in Civil Engineering, 20 (3), 217-21.

(2007), 'Modeling slump flow of concrete using second-order regressions and artificial neural networks', Cement and concrete composites, 29 (6), 474-80.

Zain, Muhammad Fauzi Mohd, et al. (2011), 'Production of rice husk ash for use in concrete as a supplementary cementitious material', Construction and building materials, 25 (2), 798-805. 